\title{
A Direct Approach for Coupling Matrix Synthesis for Coupled Resonator Diplexers
}

\author{
Deeb Tubail ${ }^{1}$, Talal Skaik ${ }^{2}$ \\ ${ }^{1}$ Palestinian Technology Research Center, Palestine, Email: dtubail@gmail.com \\ ${ }^{2}$ Electrical Engineering Department, IUG University, P.O. Box 108, Palestine, Email: talalskaik@gmail.com
}

\begin{abstract}
Diplexers are used to separate or combine two bands of frequencies and they are designed by combining channel filters using distribution circuit or alternatively designed based on coupled resonator circuits. Design parameters of coupled resonator circuits such as coupling coefficients, self-resonant frequencies and external couplings are done using optimization techniques. However, optimization techniques are not always efficient particularly for large set of parameters and hence more efficient approaches are required. In this paper, numerical solution instead of optimization is proposed to synthesize coupled resonator diplexers and obtain coupling coefficients directly from equations. The proposed direct approach achieves the desired characteristics for any given number of resonators and some numerical examples are given to demonstrate the validity of the proposed approach. One of the given examples is compared to a diplexer in literature synthesized using optimization technique and implemented using cavity resonators.
\end{abstract}

Index Terms - coupling coefficients, coupling matrix, diplexer, resonators, $\mathrm{T}$ topology.

\section{INTRODUCTION}

Diplexers play an important role in communication systems where they are used to separate or combine different bands of frequencies. Usually diplexers are consisted of two filters connected with a junction. The design of coupled resonator filters is well known in filter theory and it is based on coupling matrix that represents coupling coefficients calculated using g-values [1, 2]. Another approach to synthesize filters based on optimization techniques is also presented in literature [3]-[5]. The theory of two-port coupled resonator filters is extended to multi-port coupled resonator structures that consist of only resonators without external junctions and thus forming miniaturized diplexers and multiplexers [6]-[18]. In [7], synthesis of coupled resonator diplexers is proposed. However, the synthesis method of coupling coefficients is done by optimization techniques that may not give a solution for relatively large structures with large set of optimization variables. Many coupled resonator diplexer components synthesized using coupling matrix optimizations have been presented in literature [7]-[11]. Furthermore, coupling matrix optimization approach has been extended to all resonator multiplexers [12]-[18]. The synthesis of such components becomes more complex for large structures and optimization algorithms may not converge to give a solution. 
Fig. 1 exhibits the main coupled resonator diplexer topology presented in [7]-[10], where the coupling between the resonators, self-coupling and the external coupling coefficients are obtained using coupling matrix optimization. In this paper, we propose an alternative synthesis of coupling matrix for the topology in Fig. 1 directly from mathematical equations deduced empirically from optimization-based observations. The solution using the direct approach releases the need to use complex optimization algorithms that require defining a cost function, optimization variables and some constraints. The proposed direct synthesis achieves the desired characteristics for any number of resonators. Some numerical examples are presented in this paper for coupled resonator diplexers and an example is compared to a diplexer in literature synthesized using coupling matrix optimization.

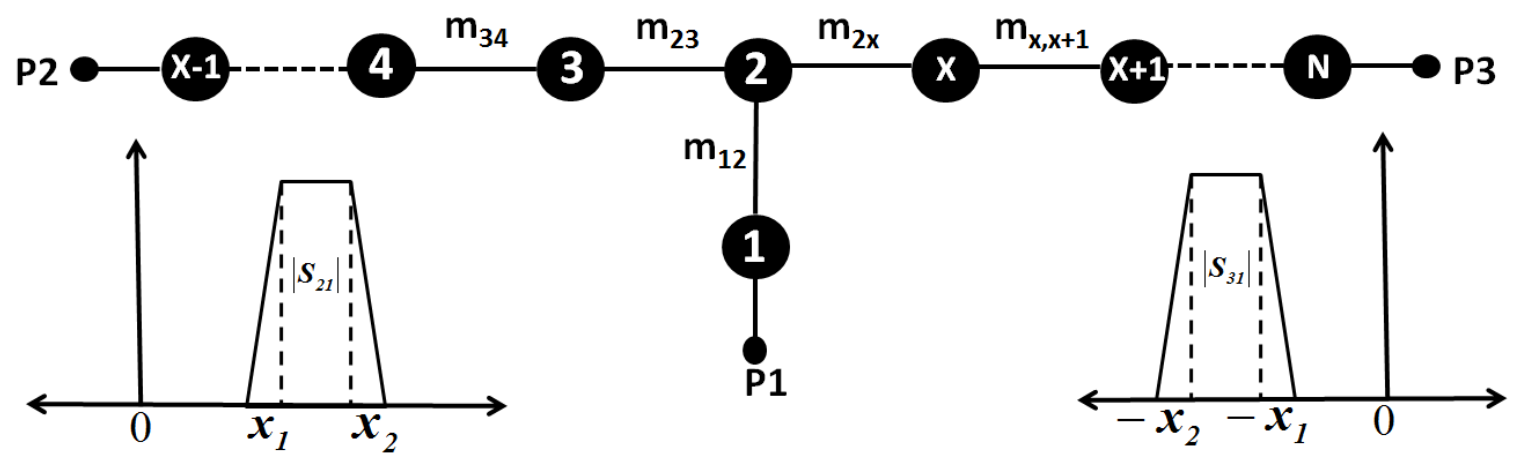

Fig. 1. Diplexer Topology.

\section{DIRECT SYNTHESIS APPROACH}

This section presents the proposed numerical solution to obtain the normalized coupling coefficients of the diplexer presented in Fig. 1 with pass-band ripple $L_{A r}=0.04321 \mathrm{~dB}$. Considering a symmetrical channel diplexer with channel 1 extending from $x_{1}$ to $x_{2}$ (normalized frequencies) and channel 2 from $-x_{2}$ to $-x_{1}$, the normalized bandwidth $B_{w}$ and the diplexer center frequency $m_{c}$ are given by

$$
\begin{gathered}
B_{w}=x_{2}-x_{1} \\
m_{v}=0.5\left(x_{1}+x_{2}\right) \\
m_{c}=\sqrt{x_{1} x_{2}}
\end{gathered}
$$

The normalized coupling coefficient $m_{12}$ is given by

$$
\begin{gathered}
a_{12}=0.9756 m_{v}^{2}-2.0877 m_{v}+1.4409 \\
b_{12}=0.0382 \\
c_{12}=-0.0048 \\
m_{12}=a_{12} B_{w}^{2}+b_{12} B_{w}+c_{12}+m_{c}
\end{gathered}
$$

where $a_{12}, b_{12}$ and $c_{12}$ are the coefficients of the quadratic equation of the coupling coefficient $m_{12}$. 
The normalized coefficient $m_{23}$ which equals to $m_{2 x}$ is given by

$$
\begin{gathered}
a_{23}=-0.0209 m_{v}+0.6939 \\
b_{23}=-1.0001 m_{v}-0.008 \\
m_{23}=a_{23} B_{w}+b_{23}+m_{c}
\end{gathered}
$$

where $a_{23}$ and $b_{23}$ are the coefficients of the linear equation of the coupling coefficient $m_{23}$. The normalized coupling coefficients between any other adjacent resonators $m_{i, i+1}$ is given by

$$
m_{i, i+1}=\frac{0.5 B_{w}}{\sqrt{g_{i} g_{i+1}}}
$$

The first two resonators $R_{1}$ and $R_{2}$ have no frequency offsets, and hence the self-coupling coefficients $m_{11}$ and $m_{22}$ are equal to zero. The self-coupling coefficient $m_{33}$ which equals $m_{x x}$ is given by

$$
\begin{gathered}
a_{33}=0.1177 \log _{10} m_{v}-0.1349 \\
b_{33}=0.0448 m_{v}-0.1777 \\
c_{33}=0.9936 m_{v}+0.0325 \\
m_{33}=a_{33} B_{w}^{2}+b_{33} B_{w}+c_{33}+m_{v}-m_{c}
\end{gathered}
$$

The self-coupling coefficients $m_{i i}$ of the other resonators in left arm $R_{i}$ where $i=4, \ldots, x$-1 are given by $m_{i i}=m_{v}$ in (1b), and similarly the other self-coupling coefficients $m_{i i}$ of other resonators in the right $\operatorname{arm} R_{i}$ where $i=x+1, \ldots, N$ are equal to $-m_{v}$ in (1b).

The external quality factor at port one $q_{e 1}$, at port two $q_{e 2}$ and at port three $q_{e 3}$ are given from [7] as

$$
\begin{gathered}
q_{e 2}=q_{e 3}=\frac{2}{B_{w}} q_{e \pm 1} \\
q_{e 1}=\frac{q_{e 2}}{2}=\frac{q_{e 3}}{2}
\end{gathered}
$$

where $q_{e \pm 1}$ is the normalized external quality factor of the filter with edges of \pm 1 , that can be calculated from the g-values as known in filter theory [1]. Finally, the scattering parameters are related to the coupling matrix as given in [7].

To illustrate how the proposed equations are deduced empirically from extensive study of optimization-based results, the following procedure is followed to give a brief insight on the foundation of the proposed method:

1- The specifications of the diplexer are firstly defined which are the total number of resonators and the edges of the channels: $x_{1}$ to $x_{2}$ for channel 1 and $-x_{2}$ to $-x_{1}$ for channel 2 .

2- The center frequency for each channel $m_{c}$ is now directly calculated by (1c) and the bandwidth $B_{w}$ by (1a).

3- Optimization procedure is utilized to synthesize the coupling coefficients using cost function 
defined in [7].

4- A comprehensive study is carried out on the effect of changing the channel bandwidth $B_{w}$ on the values of coupling coefficients.

5- Plots of optimized coupling coefficients (y-axis) versus channel bandwidth $B_{w}$ (x-axis) are obtained.

6- Step 5 is repeated for various channel positions $m_{v}$ as in (1b).

7- The coupling coefficients between adjacent resonators other than those at the junction are observed from the optimization results and they are found to be directly related to the known g-values. Fig. 2 presents an example of the optimized values of the coupling coefficients $\left(m_{i, i+1}\right)$ between two adjacent resonators other than those at the junction. Those coupling coefficients linearly change with the bandwidth of the channels $B_{w}$ and they are independent of the channel position $m_{v}$ and then (4) is deduced.

8- The self-coupling coefficients of the resonators (except resonators at the junction: $R_{1}, R_{2}, R_{3}$ and $R_{\mathrm{x}}$ ) are displayed in Fig. 3 and they are found to be independent of the bandwidth $B_{w}$ and their values are very close to the channel position $m_{v}$ where $m_{i i}=m_{v}$ is deduced.

9- The graphs of the coupling coefficients at the junction: $m_{12}, m_{23}$ and $m_{33}$ are presented in Figures 4, 5, 6 respectively. Curves in Fig. 4 represent quadratic equations for $m_{12}-m_{c}$ versus channel bandwidth where each curve is given for a certain channel position $m_{v}$. It can be noticed that the coupling coefficient $m_{12}$ is dependent on channel bandwidth $B_{w}$, channel position $m_{v}$ and center frequency $m_{c}$. Moreover, the curves representing $m_{23}-m_{\mathrm{c}}$ in Fig. 5 vary linearly with bandwidth and they are dependent on channel position $m_{v}$. Furthermore, the coefficient $m_{33}$ is depicted in Fig. 6 that exhibits quadratic equations for $m_{33}-m_{v}+m_{c}$ versus channel bandwidth $B_{w}$. It is evident from Fig. 6 that $m_{33}$ depends on $B_{w}, m_{v}$ and $m_{c}$.

10- The equations of the coupling coefficients at the junction $\left(m_{12}, m_{23}\right.$ and $\left.m_{33}\right)$ are empirically deduced from the graphs in terms of the channel bandwidth $B_{w}$ and channel position $m_{v}$.

11- The coefficients of the linear/quadratic equations which are labeled $(a, b, c)$ in equations $(2,3$, and 5) are also determined.

12- For diplexers with different number of resonators, the previous steps are repeated. Very similar results for coupling coefficients have been obtained for diplexers with different number of resonators and general equations are deduced and proposed here. 
Journal of Microwaves, Optoelectronics and Electromagnetic Applications, Vol. 16, No. 4, December 2017946 DOI: http://dx.doi.org/10.1590/2179-10742017v16i41077

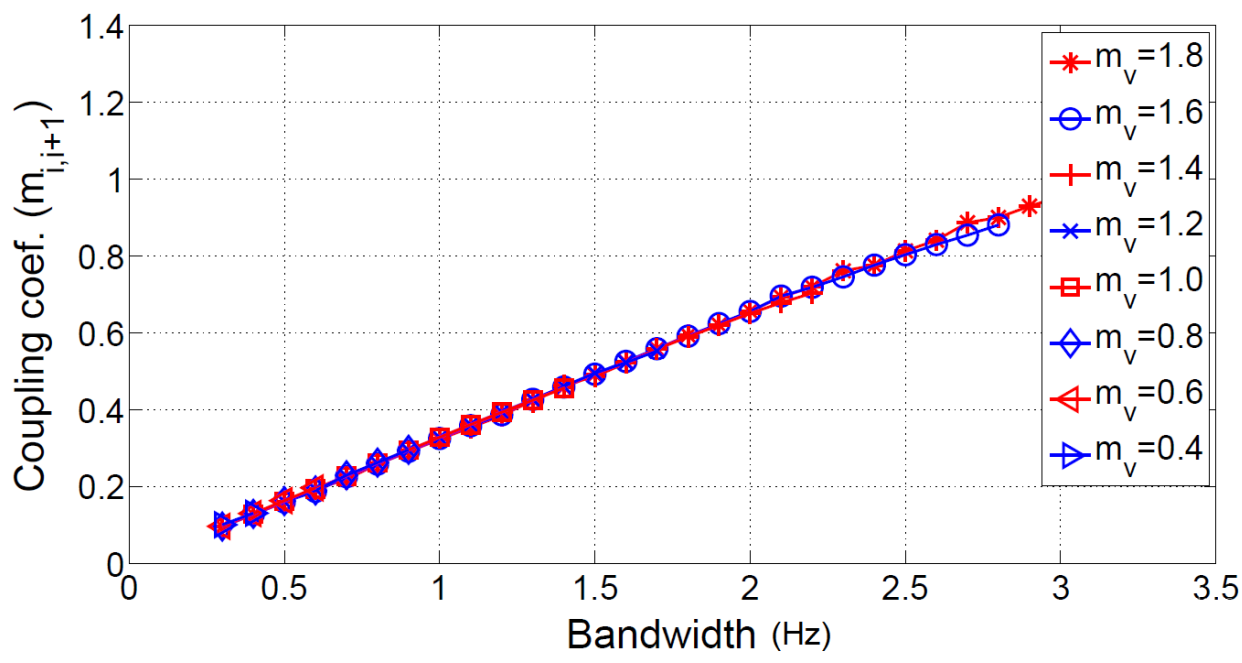

Fig. 2. Optimized values of coupling coefficient $m_{i, i+1}$.

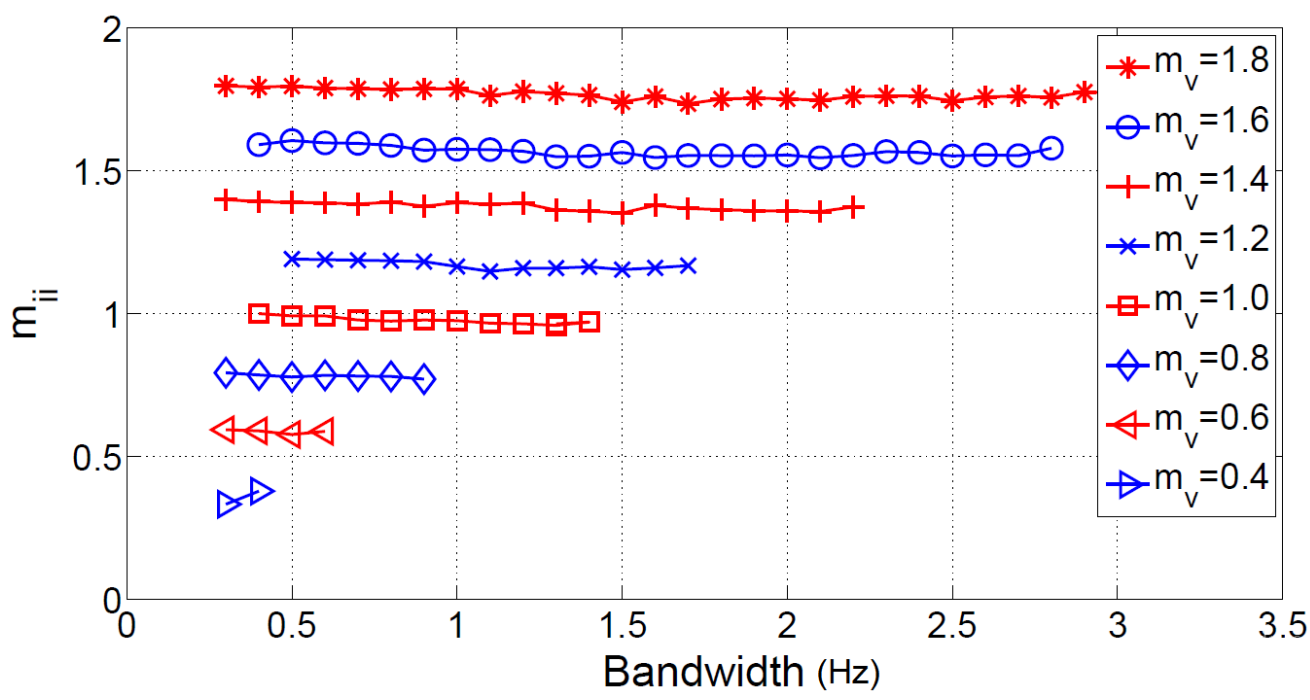

Fig. 3. Optimized values of coupling coefficient $m_{i i}$.

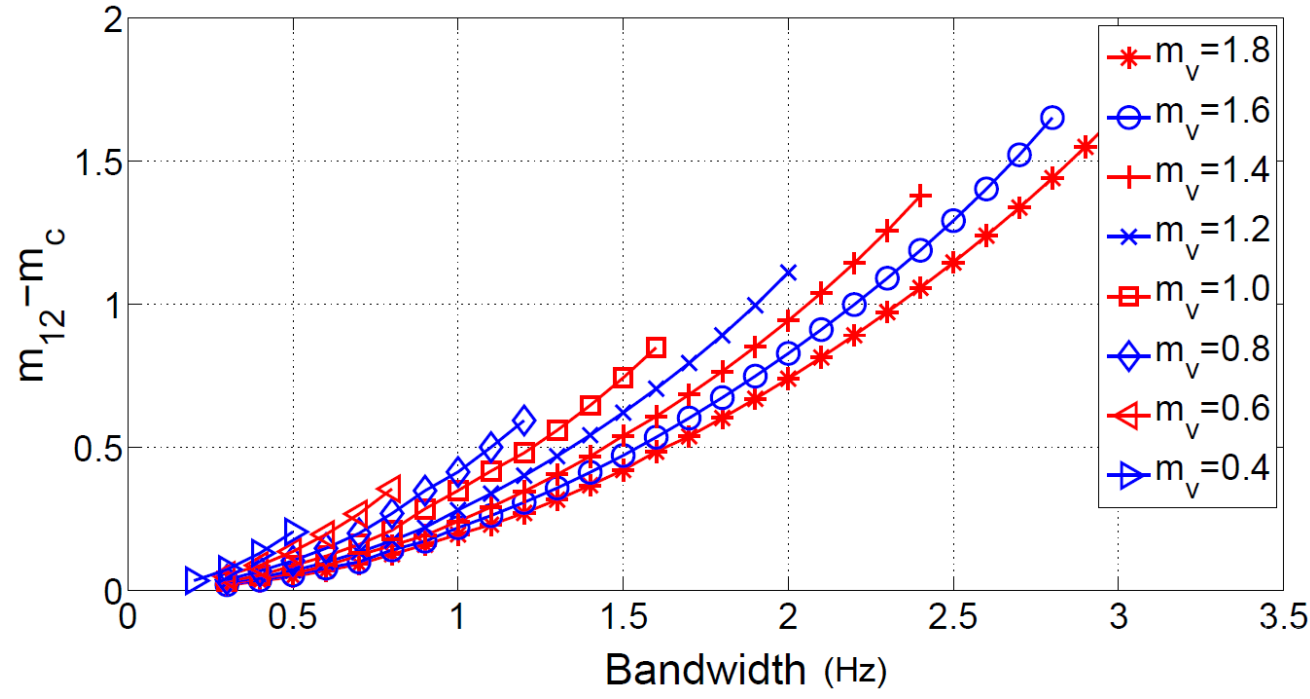

Fig. 4. Optimized values of coupling coefficient $m_{12}$. 


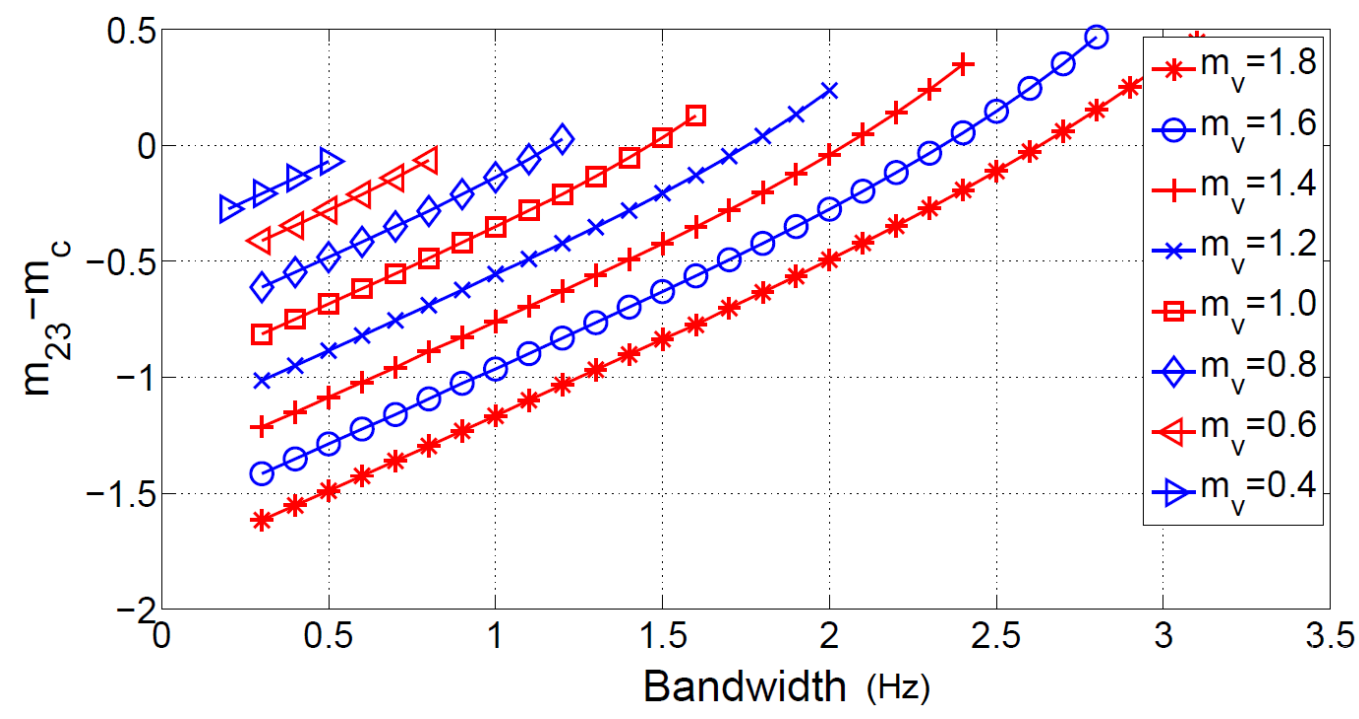

Fig. 5. Optimized values of coupling coefficient $m_{23}$.

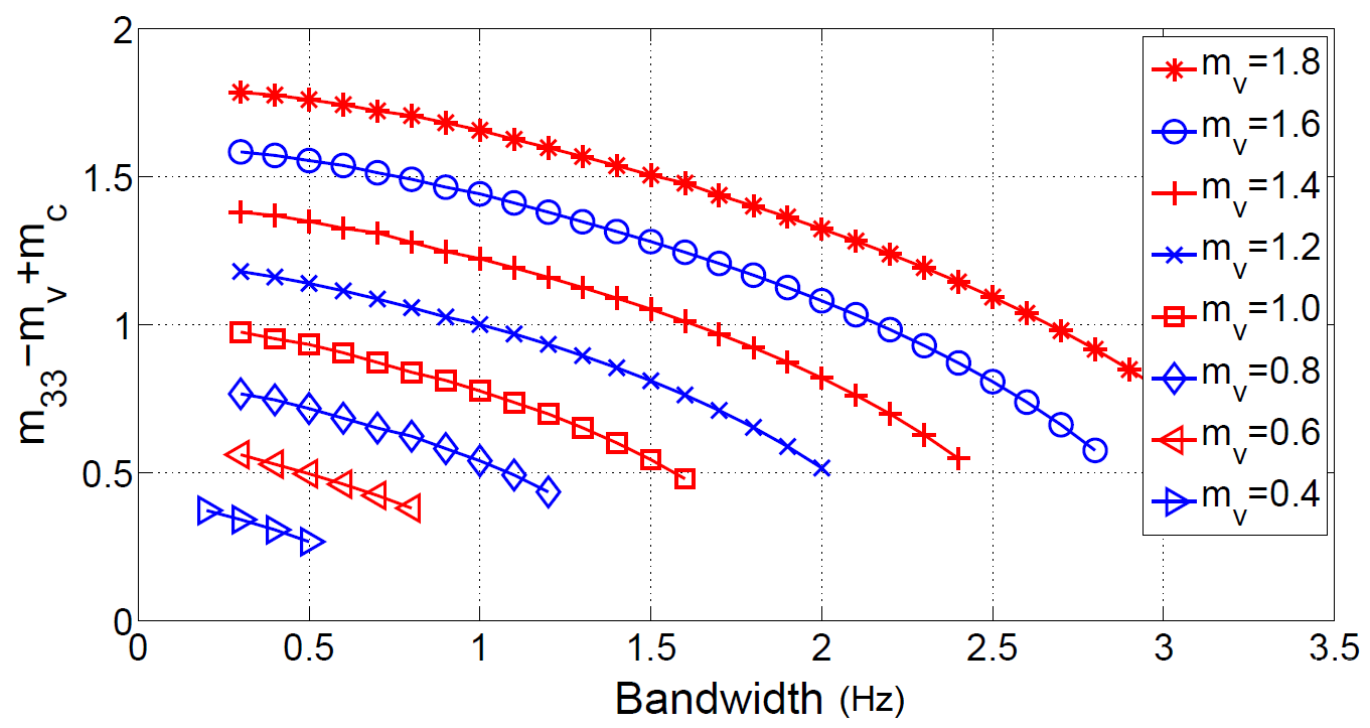

Fig. 6. Optimized values of coupling coefficient $m_{33}$.

\section{NUMERICAL EXAMPLES}

This section presents numerical examples for coupled resonator diplexers synthesized using proposed equations in previous section. Five examples are given to prove the ability of the direct method to satisfy various diplexers' characteristics and requirements. These examples are different in number of resonators as well as different bandwidths. One of the examples is synthesized using direct method and compared with a diplexer in a literature synthesized using optimization and implemented using waveguide cavities.

Fig. 7 presents the topology of example one which consists of ten resonators. The normalized edges of the channels are $x_{1}=0.3, x_{2}=1$ and the normalized calculated coupling coefficients are $m_{12}=0.8127$, $m_{23}=m_{27}=0.3659, \quad m_{34}=m_{78}=0.2226, \quad m_{45}=m_{89}=0.2226$ and $m_{56}=m_{9,10}=0.3032$. The self-coupling coefficients are $m_{11}=m_{22}=0, m_{33}=-m_{77}=0.5857, m_{44}=m_{55}=m_{66}=-m_{88}=-m_{99}=-m_{10,10}=0.65$. The normalized 
external quality factor of port two and port three is $q_{\mathrm{e} 2}=q_{e 3}=2.7754$, while the normalized external quality factor of the common port is $q_{e 1}=1.3877$. The diplexer response is presented in Fig. 8 and it can be seen that the desired diplexer specification is achieved with five reflection zeros for each channel and with the specified channel edges and return loss.

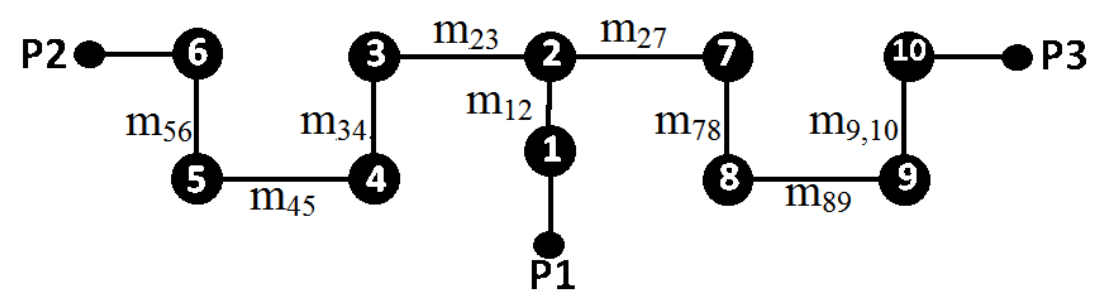

Fig. 7. Topology of example one.

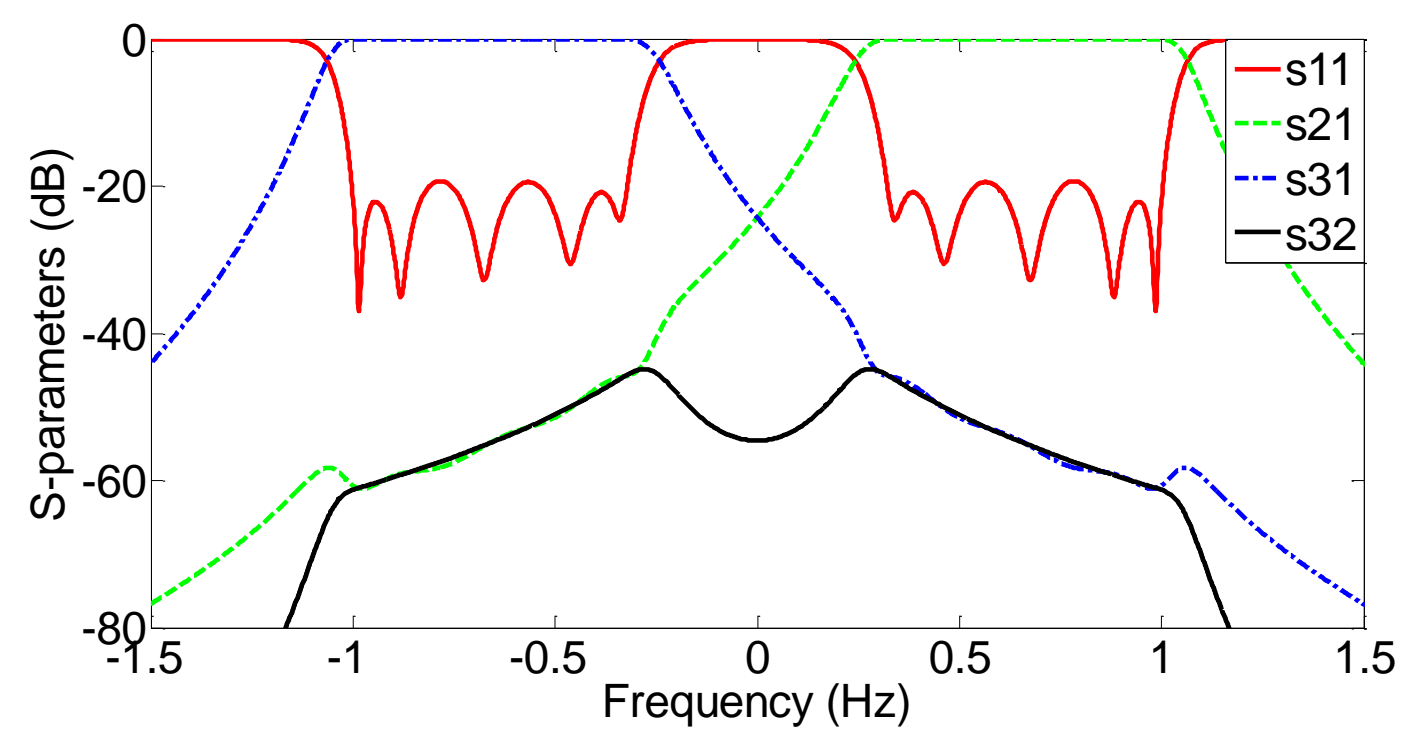

Fig. 8. Theoretical response of example one.

Topology in Fig. 9 presents the diplexer of example two of sixteen resonators. The normalized edges of the channels are $x_{1}=0.15, x_{2}=1$ and the normalized coupling coefficients are $m_{12}=0.8218$, $m_{23}=m_{2,10}=0.3838, \quad m_{34}=m_{10,11}=0.2515, \quad m_{45}=m_{11,12}=0.2354, \quad m_{56}=m_{12,13}=0.2321, \quad m_{67}=m_{13,14}=0.2354$, $m_{78}=m_{14,15}=0.2515$ and $m_{89}=m_{15,16}=0.3498$. The self-coupling coefficients are $m_{11}=m_{22}=0, m_{33}=-m_{10,10}=$ $0.5178, m_{44}=m_{55}=m_{66}=m_{77}=m_{88}=m_{99}=-m_{11,11}=-m_{12,12}=-m_{13,13}=-m_{14,14}=-m_{15,15}=-m_{16,16}=0.5750$. The normalized external quality factor of port two and port three is $q_{e 2}=q_{e 3}=2.3932$, while the normalized external quality factor of the common port is $q_{e 1}=1.1966$. The response of the diplexer is shown in Fig. 10 and it can be noticed that eight reflection zeros appear in each channel and that the specified channel bandwidth is satisfied.

Example three is a diplexer formed of twenty coupled resonators and its topology is depicted in Fig. 11. The values of the boundaries are $x_{1}=0.12$ and $x_{2}=1$ and the calculated normalized external quality 
factors are $q_{e 2}=q_{e 3}=2.3364$ and $q_{e 1}=1.1682$. The calculated normalized coupling coefficients are $m_{12}=0.8226, \quad m_{23}=m_{2,12}=0.3787, \quad m_{34}=m_{12,13}=0.2570, \quad m_{45}=m_{13,14}=0.2396, \quad m_{56}=m_{14,15}=0.2342$, $m_{67}=m_{15,16}=0.2328, \quad m_{78}=m_{16,17}=0.2342, \quad m_{89}=m_{17,18}=0.2396, \quad m_{9,10}=m_{18,19}=0.2570, \quad$ and $m_{10,11}=m_{19,20}=0.3580$. The self-coupling coefficients are $m_{11}=m_{22}=0, \quad m_{33}=-m_{12,12}=0.5109$, $m_{44}=m_{55}=m_{66}=m_{77}=m_{88}=m_{99}=m_{10,10}=m_{11,11}=-m_{13,13}=-m_{14,14}=-m_{15,15}=-m_{16,16}=-m_{17,17}=-m_{18,18}=-m_{19,19}=-m_{20,20}$ $=0.5600$. The diplexer response is presented in Fig. 12 and it is evident that the specification of the diplexer is met which indicates that the proposed direct synthesis method is robust and convenient for large topologies. If this diplexer is synthesized using optimization techniques, it requires more than twenty variables with some constraints which may complicate the optimization problem and make the solution infeasible.

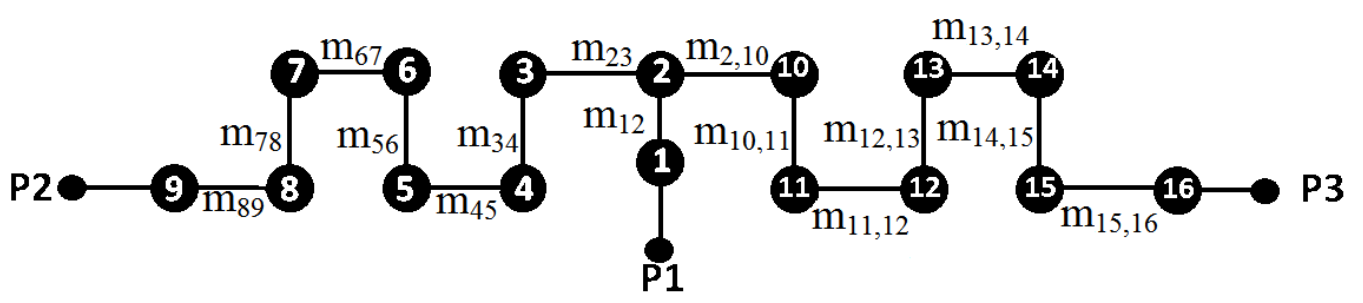

Fig. 9. Topology of example two.

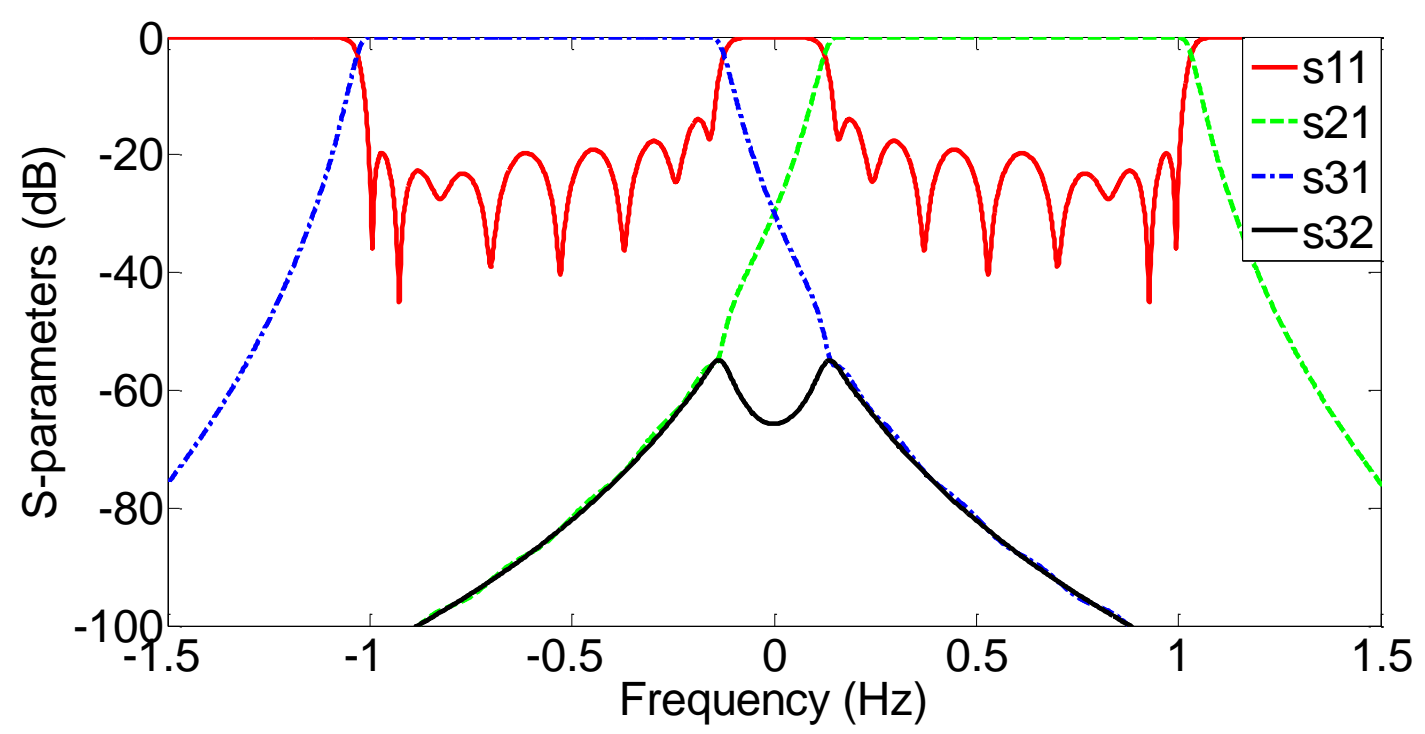

Fig. 10. Theoretical response of example two.

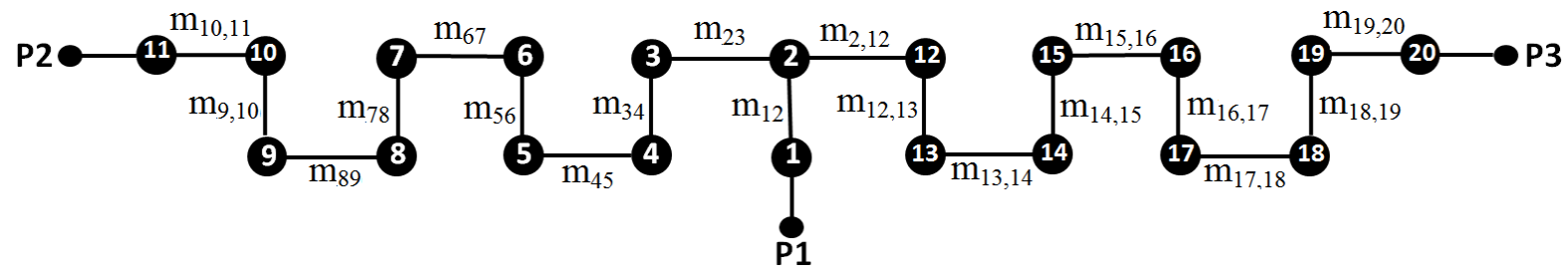

Fig. 11. Topology of example three. 


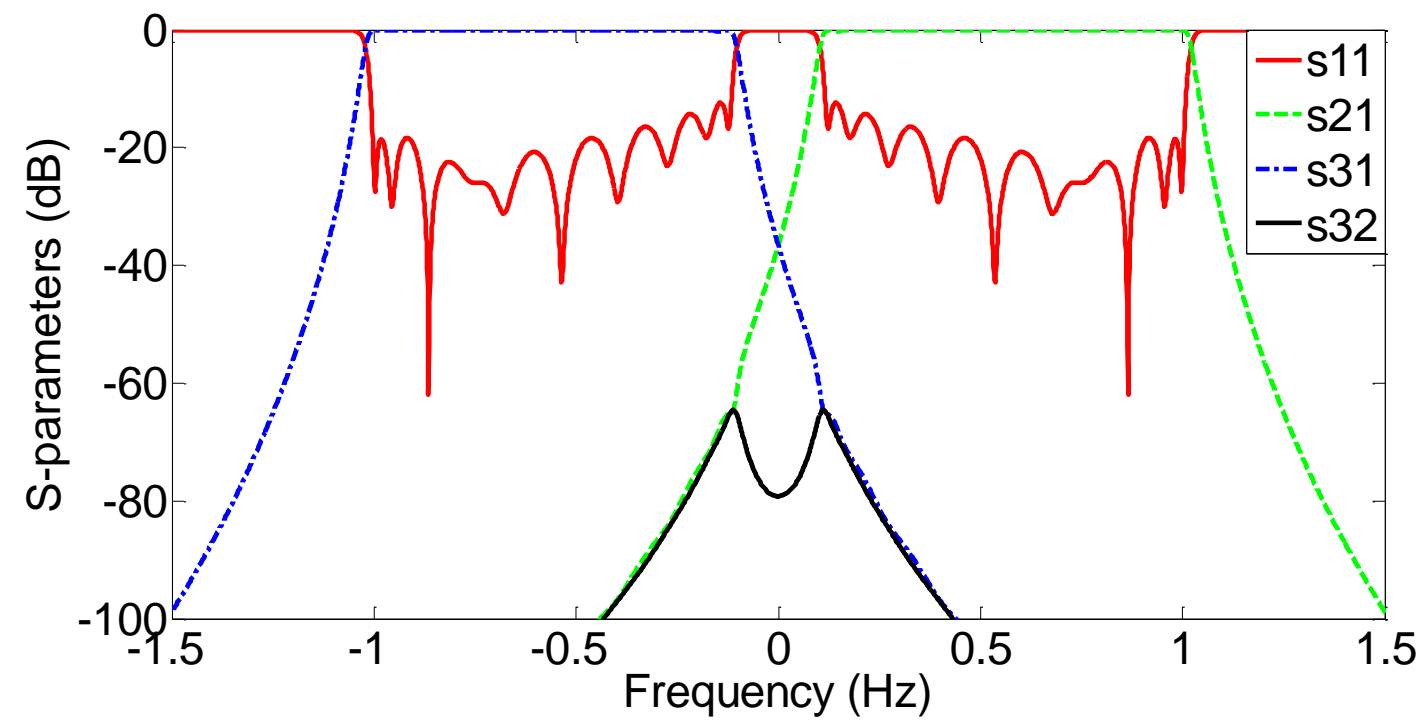

Fig. 12. Theoretical response of example three.

Example four presents a diplexer with twelve coupled resonators and its topology is shown in Fig. 13. The specifications of this diplexer are the same as those in [9] that is practically implemented using waveguide cavity resonators at $10 \mathrm{GHz}$. The values of the boundaries are $x_{1}=0.30618$ and $x_{2}=1$ and the calculated normalized external quality factors are $q_{e 2}=q_{e 3}=2.9806$ and $q_{e 1}=1.4903$. A comparison between coupling coefficients for both methods is shown in Table I that clearly shows very close results for both methods. The response of diplexer four is depicted in Fig. 14 for both the direct and optimization techniques. It can be seen from the graph that the plots of S-parameters are close for both the proposed direct method and the optimization-based synthesis approach. The optimization has been performed on computer with Intel processor core i7, a CPU at $2.4 \mathrm{GHz}$ and a RAM of 8 GB.

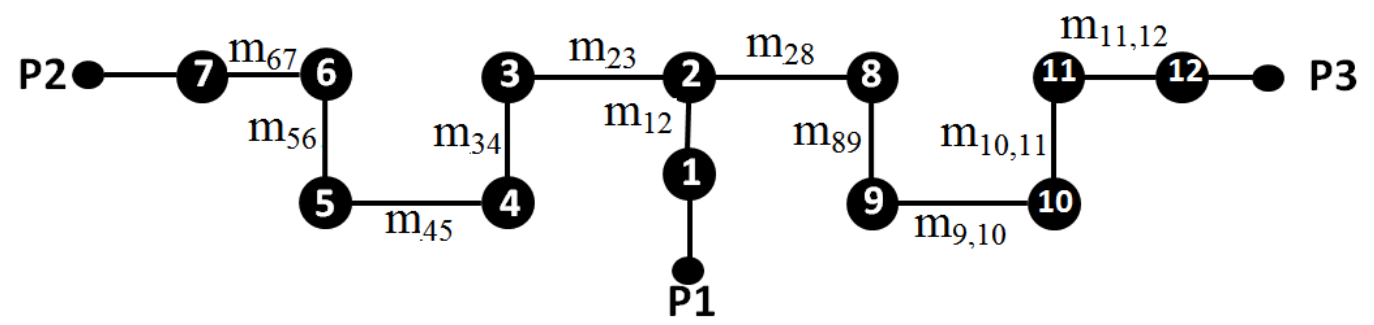

Fig. 13. Topology of example four.

Example five presents a diplexer with fourteen coupled resonators and its topology is shown in Fig. 15. The values of the boundaries are $x_{1}=0.2$ and $x_{2}=1$ and the calculated normalized external quality factors are $q_{e 2}=q_{e 3}=2.52$ and $q_{e 1}=1.26$. The coupling coefficients synthesized by both direct and optimization methods are compared in Table II where it can be noticed that the results are close. Fig. 16 presents the response of the diplexer for both methods where small differences are observed. 


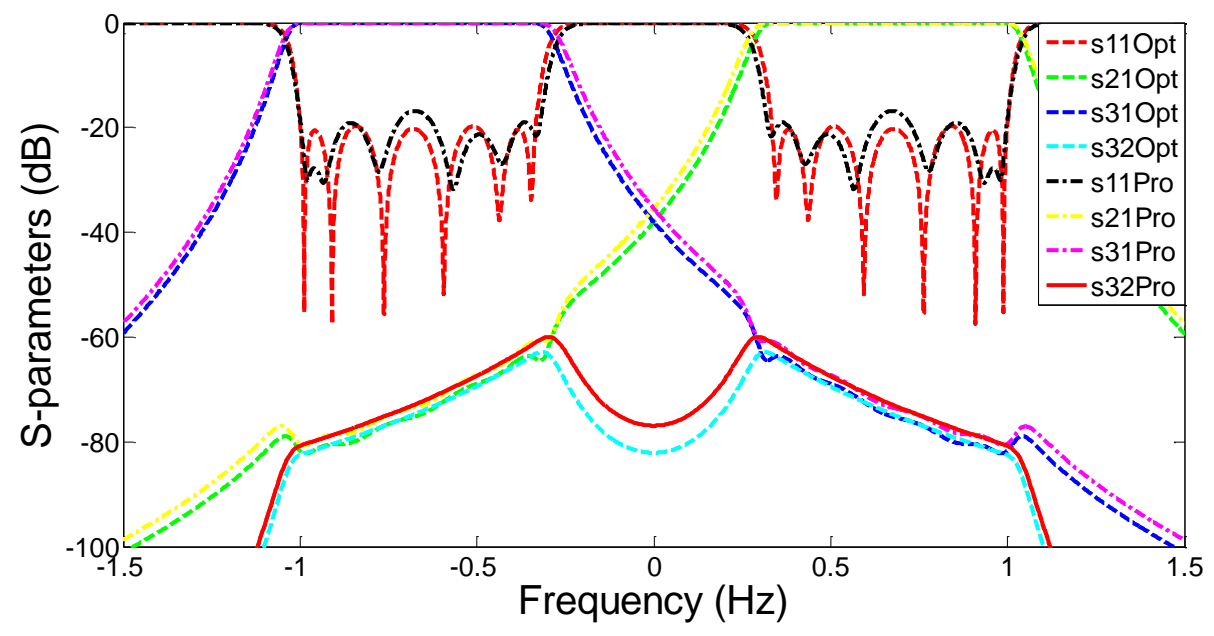

Fig. 14. Theoretical response of example four.

TABLE I. NORMALIZED COUPLING COEFFICIENTS OF EXAMPLE FOUR

\begin{tabular}{|c|c|c|}
\hline Norm. Coupling Coef. & Proposed Method & Optimization Method \\
\hline$m_{12}$ & 0.8125 & 0.7963 \\
\hline$m_{23}=m_{28}$ & 0.3560 & 0.3466 \\
\hline$m_{34}=m_{89}$ & 0.2039 & 0.2101 \\
\hline$m_{45}=m_{9,10}$ & 0.1946 & 0.195 \\
\hline$m_{56}=m_{10,11}$ & 0.2039 & 0.2035 \\
\hline$m_{67}=m_{11,12}$ & 0.2814 & 0.2814 \\
\hline$m_{11}=m_{22}$ & 0 & 0 \\
\hline$m_{33}=-m_{88}$ & 0.6043 & 0.5942 \\
\hline$m_{44}=-m_{99}$ & 0.6665 & 0.655 \\
\hline$m_{55}=-m_{10,10}$ & 0.6665 & 0.6635 \\
\hline$m_{66}=-m_{11,11}$ & 0.6665 & 0.6652 \\
\hline$m_{77}=-m_{12,12}$ & 0.6665 & 0.6643 \\
\hline "Time consumption & Instant calculations & $\begin{array}{l}\text { 47.0782 second optimization } \\
\text { time }+ \text { time of writing } \\
\text { optimization code. }\end{array}$ \\
\hline Complexity & $\begin{array}{l}\text {-No complexity, } \\
\text { direct calculations } \\
\text { from equations }\end{array}$ & $\begin{array}{l}\text {-Complex cost function [see } \\
\text { equation (3) in [9]. } \\
\text {-Large number of } \\
\text { optimization variables (17 } \\
\text { variables). }\end{array}$ \\
\hline
\end{tabular}

The results of those previous examples verify that the proposed numerical equations achieve the desired characteristics for different number of resonators and different channel bandwidths for coupled resonator diplexer with T-topology. Moreover, the proposed method is preferred to optimization techniques presented in literature for the given T-topology since the coupling coefficients are directly calculated from equations. 
Journal of Microwaves, Optoelectronics and Electromagnetic Applications, Vol. 16, No. 4, December 2017952 DOI: http://dx.doi.org/10.1590/2179-10742017v16i41077

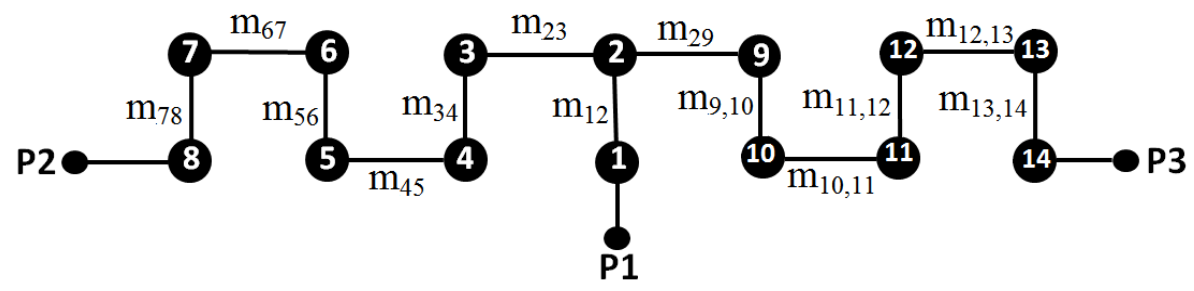

Fig. 15. Topology of example five.

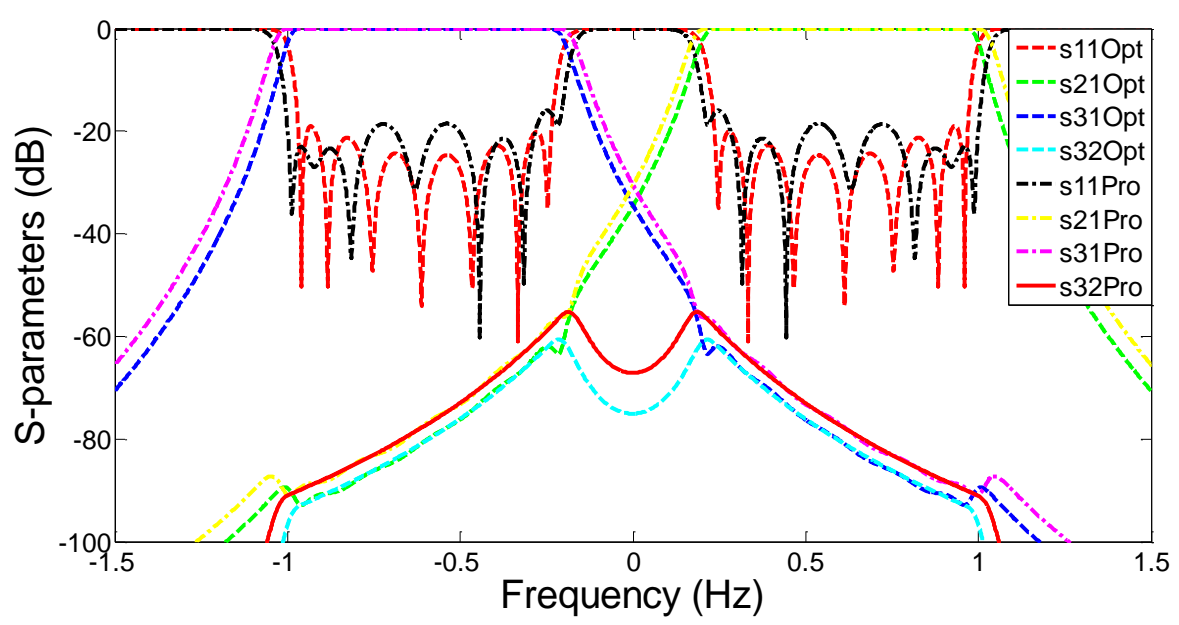

Fig. 16. Theoretical response of example five.

TABLE II. NORMALIZED COUPLING COEFFICIENTS OF EXAMPLE FIVE

\begin{tabular}{|c|c|c|}
\hline Norm. Coupling Coef. & Proposed Method & Optimization Method \\
\hline$m_{12}$ & 0.8183 & 0.7790 \\
\hline$m_{23}=m_{29}$ & 0.3842 & 0.3646 \\
\hline$m_{34}=m_{9,10}$ & 0.2396 & 0.2304 \\
\hline$m_{45}=m_{10,11}$ & 0.2255 & 0.2165 \\
\hline$m_{56}=m_{11,12}$ & 0.2255 & 0.2146 \\
\hline$m_{67}=m_{12,13}$ & 0.2396 & 0.2242 \\
\hline$m_{78}=m_{13,14}$ & 0.3324 & 0.3162 \\
\hline$m_{11}=m_{22}$ & 0 & 0 \\
\hline$m_{33}=-m_{99}$ & 0.5360 & 0.5188 \\
\hline$m_{44}=-m_{10,10}$ & 0.6000 & 0.5877 \\
\hline$m_{55}=-m_{11,11}$ & 0.6000 & 0.6022 \\
\hline$m_{66}=-m_{12,12}$ & 0.6000 & 0.5953 \\
\hline$m_{77}=-m_{13,13}$ & 0.6000 & 0.5928 \\
\hline$m_{88}=-m_{14,14}$ & 0.6000 & 0.5950 \\
\hline Time consumption & Instant calculations & $\begin{array}{l}328.5 \text { second optimization } \\
\text { time }+ \text { time of writing } \\
\text { optimization code }\end{array}$ \\
\hline Complexity & $\begin{array}{l}\text {-No complexity, } \\
\text { direct calculations } \\
\text { from equations }\end{array}$ & $\begin{array}{l}\text {-Complex cost function [see } \\
\text { equation (3) in [9]. } \\
\text {-Large number } \\
\text { optimization variables } \\
\text { variables). }\end{array}$ \\
\hline
\end{tabular}




\section{CONCLUSION}

This paper presents a novel direct approach for synthesis of coupled resonator diplexers with Ttopology. The proposed method is based on synthesis of coupling coefficients directly from equations instead of optimization. The direct method achieves the desired diplexer characteristics for different number of resonators and various bandwidth requirements. An illustration on deducing the proposed equations from comprehensive optimization results is presented to give a good insight about the basis of the method. Five examples are given to prove the validity of the approach for relatively large number of resonators. Moreover, one numerical example is presented and compared to a diplexer synthesized and implemented in previous literature. The proposed method beats the optimization technique in reducing time consumption and complexity for structures with high number of resonators.

\section{REFERENCES}

[1] J. Hong, M. Lancaster, Microstrip Filters for RF/Microwave Applications, Wiley Interscience Publication, New York, 2001.

[2] D. Pozar, Microwave Engineering, $4^{\text {th }}$ edition, Wiley, 2012.

[3] S. Amari, "Synthesis of cross-coupled resonator filters using an analytical gradient-based optimization technique," IEEE Trans. Microw. Theory Tech, vol. 48, no. 9, pp. 1559-1564, 2000.

[4] A. Atia, A. Zaki, E. Atia, "Synthesis of general topology multiple coupled resonator filters by optimization," in Proc. of IEEE MTT-S Int. Microwave Symposium, Baltimore, USA, 1998, pp. 821-824.

[5] B. Jayyousi, M. Lancaster, "A gradient-based optimization technique employing determinants for the synthesis of microwave coupled filters," in Proc. of IEEE MTT-S Inter. Microwave Symposium, USA, June 2004, pp. 1369-1372.

[6] M. Lancaster, "Radio frequency filter", W.I.P.O patent WO/01/69712, 2001.

[7] T. Skaik, M. Lancaster, F. Huang, "Synthesis of multiple output coupled resonator microwave circuits using coupling matrix optimization," IET Journal of Microwaves, Antenna and Propagation, vol. 5, no. 9, pp. 1081-1088, June 2011.

[8] T. Skaik, T., M. AbuHussain, "Design of diplexers for E-band communication systems," in Proc. of the $13^{\text {th }}$ Mediterranean Microwave Symposium, Lebanon, Sept. 2013.

[9] T. Skaik, M. Lancaster, "Coupled resonator diplexer without external junction," Journal of Electromagnetic Analysis and Applications, vol. 3, no. 6, pp. 238-241, June 2011.

[10] T. Skaik, M. Lancaster, M. Ke, Y. Wang, "A micro-machined WR-3 waveguide diplexer based on coupled resonator structures," in Proc. of the $41^{\text {st }}$ European Microwave Conference, UK, Oct 2011, pp. 770-773.

[11] W. Xia, X. Shang, M. Lancaster, "All-resonator-based waveguide diplexer with cross-couplings," in Electronics Letters, vol. 50, No. 25, pp. 1948-1950, 2014.

[12] F. Loras-Gonzalez, I. Hidalgo-Carpintero, S. Sobrino-Arias, A. Garca-Lamprez, M. Salazar-Palma, "A novel Ku-Band dielectric resonator triplexer based on generalized multiplexer theory," in Proc. of IEEE MTT-S International Microwave Symposium, 2010, Anaheim, CA, 2010, pp. 884-887.

[13] T. Skaik, "Novel star junction coupled resonator multiplexer structures," Progress in Electromagnetics Research Letters, vol. 31, pp. 113-120, April 2012.

[14] D. Tubail, T. Skaik, "Synthesis of coupled resonator based multiplexers with generalized structures using coupling matrix optimization," IET Electronic Letters, vol. 51, no. 23, pp. 1891-1893, Nov 2015.

[15] A. Garcia-Lamperez, M. Salazar-Palma, T. Sarkar, "Compact multiplexer formed by coupled resonators with distributed coupling," in Proc. IEEE Antennas and Propagation Society International Symposium, USA, 2005, pp. 89-92.

[16] T. Skaik, D. Tubail, "Novel multiplexer topologies based on coupled resonator structures," in Proc. of the $15^{\text {th }}$ Mediterranean Microwave Symposium, Lecce, Italy, Dec. 2015.

[17] X. Shang, Y. Wang, X. Wenlin, M. Lancaster, "Novel multiplexer topologies based on all-resonator structures," IEEE Transactions Microwave Theory and Techniques, vol. 61, no. 11, pp. 3838-3845, 2013.

[18] Y. Wang, M. Lancaster, "An investigation on the coupling characteristics of a novel multiplexer configuration," in Proc. of European Microwave Conference (EuMC), Nuremberg, 2013, pp. 900-903. 